\title{
Retracted: Optimization Method of Sports Service Network Node Layout Based on Network Communication
}

\author{
Security and Communication Networks
}

Received 7 April 2022; Accepted 7 April 2022; Published 17 May 2022

Copyright ( 2022 Security and Communication Networks. This is an open access article distributed under the Creative Commons Attribution License, which permits unrestricted use, distribution, and reproduction in any medium, provided the original work is properly cited.

Security and Communication Networks has retracted the article titled "Optimization Method of Sports Service Network Node Layout Based on Network Communication” [1], due to concerns with the authenticity of the data. It was found that previous versions of this submission contained a figure unrelated to the topic of the paper and this graph was subsequently identified within several other submissions, all with accompanying text claiming to have generated the graph. A number of these submissions were rejected from the journal; however, 6 were published and have now been retracted from Security and Communication Networks and Scientific Programming [2-6].

The authors responded to explain that an author from one of the identified submissions had provided copy editing for their manuscript and introduced the graph and accompanying text in error.

The authors were unable to provide copies of correspondence to support their claim or the raw data from their study. The authors' explanation did not satisfy the concerns of the editorial board, and the article is therefore being retracted due to concerns with the reliability of the data.

The authors do not agree to the retraction.

\section{References}

[1] G. Yin, R. Qi, J. Zhao, X. Zhao, and J. Xu, "Optimization Method of Sports Service Network Node Layout Based on Network Communication," Security and Communication Networks, vol. 2021, Article ID 3013135, 8 pages, 2021.

[2] L. Yan, "Research on Crude Oil Trade Procurement Model based on DEA-Malmquist Algorithm," Scientific Programming, vol. 2021, Article ID 6360439, 10 pages, 2021.

[3] L. Fan, M. Xia, P. Huang, and J. Hu, "Research on Educational Information Platform Based on Cloud Computing," Security and Communication Networks, vol. 2021, Article ID 3109473, 11 pages, 2021.

[4] L. Huo, "Intelligent Recognition Method of Vehicle Path with Time Window Based on Genetic Algorithm," Security and Communication Networks, vol. 2021, Article ID 3614291, 11 pages, 2021.

[5] L. Keshun, Z. Xijun, and Z. Xing, "Research on Analysis and Classification of Vulnerability of Electromagnetic Pulse with a STM32 Single-Chip Microcomputer," Scientific Programming, vol. 2021, Article ID 6836158, 10 pages, 2021.

[6] L. Zhuang, Y. Qi, and F. Zhang, "Braking Control System of Oilfield Minor Repair Machine Based on Wireless Sensor Network," Security and Communication Networks, vol. 2021, Article ID 6966041, 14 pages, 2021. 


\title{
Optimization Method of Sports Service Network Node Layout Based on Network Communication
}

\author{
Guang Yin, Ruiming Qi ${ }^{\mathbb{D}}$, Jinixiang Zhao, Xiaodong Zhao, and Jingchao Xu
}

Sports Department, Hebei Academy of Fine Arts, Shijiazhuang, Hebei 050700, China

Correspondence should be addressed to Ruiming Qi; yg@hbafa.edu.cn

Received 20 August 2021; Revised 13 September 2021; Accepted 19 October 2021; Published 29 October 2021

Academic Editor: Jian Su

Copyright (c) 2021 Guang Yin et al. This is an open access article distributed under the Creative Commons Attribution License, which permits unrestricted use, distribution, and reproduction in any medium, provided the original work is properly cited.

\begin{abstract}
Aiming at the problems of traditional sports service network node layout optimization methods, which have low node coverage, node moving distance, and long distance, a sports service network node layout optimization method based on network communication is proposed. The approximate algorithm is used to calculate the probability that the wireless communication network covers the sports service network node. According to the energy consumption generated by the sports service network node in the communication process and the energy generated by the node transmission data, the energy consumption model is constructed to obtain all the communication nodes of the sports service network. Taking the maximum coverage of the rectangular area of the sports service network communication node and the minimum energy consumption of the node as the optimization goal, the accelerated genetic algorithm is used to construct the objective function to optimize the layout of the sports service network node. The simulation experiment results show that the sports service network node coverage of the proposed method has a wide coverage and a shorter node moving distance, which can effectively extend the life of the sports service network.
\end{abstract}

\section{Introduction}

The advent of the Internet age has had a major impact on our production and life. At the same time, the Internet has provided a powerful driving force for the development of our country. My country is currently striving to implement the "Internet +" strategy and actively promote the deep integration of various fields with the Internet. The sports service network platform is the integration of sports and the Internet, and it is a product that conforms to the times [1]. On the one hand, the sports industry can use the network platform to add vitality to the operation of the stadium; on the other hand, through the use of Internet technology, it can provide ordinary people with a better sports experience so that everyone can fully experience the charm of sports. Sports authorities can also use sports network platforms to revitalize stadium resources and provide services for public fitness. People can also fully enjoy low-priced sports public services [2]. Since the center of the sports service network node transmitting and receiving data is called the base station, the method of the node with a shorter distance from the base station and the larger scale of data transmission leads to the end of use of the node early, which affects the service life and performance of the sports service network. Therefore, in the sports service network, effectively laying out the nodes of the sports service network is the key way to expand the scope of monitoring and prolong the service life of the network [3].

The authors of [4] propose a multinode communication channel optimization method based on the hierarchical layout method, use the vector communication routing protocol to search for relevant hierarchical layout parameters, and calculate the distribution weight spectrum characteristics of the communication nodes to complete the communication node based on the hierarchical layout method. Allocation, on this basis, by adjusting the physical layer frame format and determining the accuracy of channel transmission time, the fixed-point processing of the multinode communication data link is achieved, and the establishment of the multinode communication channel optimization method based on the hierarchical layout method is completed. Chen et al. [5] proposed a method for optimizing the layout of the wireless sensor network nodes in 
the dam corridor. Improving the LEACH protocol analyzed the strategy and layout method of the wireless sports service network nodes in the corridor, which will affect the cluster head election. The research objects are the distance between nodes, the remaining energy of the nodes, and the distance between the nodes and the base station. The cluster head election strategy is studied, and a weight-based wireless sports service network node layout method is proposed. By calculating the proportion of each factor, the election formula is optimized. Select the most reasonable cluster head node. However, the network node layout effect of the above two methods is poor, and the coverage rate of the network node is low. Yin et al. [6] proposed a parallel computing architecture-based optimization layout method for watershed precipitation wireless sensor networks. Using this method, the rationality of the current station network in the middle and lower reaches of the Yalong River was analyzed, and the station optimization layout was studied. The results showed that based on the optimized layout method of regression, Kriging can reasonably optimize the layout of the precipitation wireless sensor network. Liu [7] proposed a method for locating congested nodes in a mobile network under the Internet of Things based on adaptive information fusion tracking and detection, building a communication transmission channel model between mobile network nodes under the Internet of Things, and using the carrier modulation method to estimate the characteristic parameters of the mobile network channel. Construct a node distance function based on the attributes of the congested node in the mobile network, and use the congested node distance calculation residual correction model to perform a nonlinear transformation on the obtained rough congested node location data to ensure the accuracy of congested node location. However, the above two methods cannot reasonably allocate the movement of each sports service network node, which leads to confusion in the movement path. Aiming at the problems of the above methods, this paper proposes a method for optimizing the layout of sports service network nodes based on network communication.

The main research innovations of the thesis are as follows:

(1) We proposed a sports service network node layout optimization method based on network communication

(2) The approximate algorithm is used to calculate the probability that the sports service network node is covered by the wireless communication network

(3) According to the energy consumption generated by the sports service network node in the communication process and the energy generated by the node transmission data, the energy consumption model is constructed to obtain all the communication nodes of the sports service network

In Section 2, we discuss the optimization method of sports service network node layout based on network communication. The simulation experiment analysis is presented in Section 3. Section 4 gives the conclusion of the article.

\section{Optimization Method of Sports Service Network Node Layout Based on Network Communication}

2.1. Sports Service Network Coverage Based on Network Communication. Due to the underdevelopment of the sports service network, the signal transmitted by the communication node will be affected by irresistible factors. After the communication wave propagates through a path, the energy will be attenuated due to the diffusion of the radiated energy [8]. Combined with the principle of radio wave propagation loss in free space, it is assumed that the sports service network communication node works in a rectangular area composed of several subregions and comprehensively considers the attenuation caused by the spread of radiant energy during the propagation process and the regional environmental factors. The attenuation of its comprehensive attenuation can be defined as

$$
L=L_{0}+\delta_{0}
$$

where $L_{0}$ is the free space loss and $\delta_{0}$ is the environmental influence factor.

The wireless communication network covers the sports service network node. If the received power $P_{r}$ of the node satisfies

$$
P_{r}=P_{t}-L \geq P_{q},
$$

where $P_{t}$ is the transmitting power of the transmitting node; $P_{q}$ is the receiving power threshold of the receiving node, through the above formula, the maximum transmission radius $R_{e}$ of the communication node can be reversed. It represents the distance at which the data packet can be successfully transmitted, which is mainly determined by the wireless propagation characteristics such as the transmission power and the intermediate frequency of the used wireless frequency [9]. The expression is

$$
R_{e}=10\left(P_{t}-P_{q}-201 g f-32.44-\delta\right) / 20 .
$$

This paper uses an approximate algorithm to calculate the coverage of the sports service network. It is assumed that the rectangular area is divided into $m \times m$ grid elements, the center of the grid element is $G\left(X_{j}, Y_{k}\right)$, the area of each grid element is $\Delta S$, the total area of the rectangular area is $S$, and the communication node layout in the center of the grid element and the event that the wireless communication node covers the grid element center $i$ is defined as $R_{i}$. Then, the probability $p\left\{R_{i}\right\}$ of the occurrence of the event is the probability $p\left(X_{j}, Y_{k}, i\right)$ that the grid element center $G\left(X_{j}, Y_{k}\right)$ is covered by the sports service network communication node $i$, which is

$$
p\left\{R_{i}\right\}=p\left(X_{j}, Y_{k}, i\right)= \begin{cases}1, & \sqrt{\left(X_{j}-X_{i}\right)^{2}+\left(Y_{k}-Y_{i}\right)^{2} \leq R_{e}}, \\ 0, & \text { otherwises. }\end{cases}
$$

This shows that if the distance between a grid element center $G\left(X_{j}, Y_{k}\right)$ and the sports service network communication node $i$ is less than or equal to the node's maximum 
transmission radius $R_{e}$, then the sports service network center is considered to be covered by the communication node $i[10]$.

Assuming that all random events $R_{i}$ are independent of each other, that is, $R_{j}$ and $R_{k}$ are independent, and $j \neq k$, then

$$
p\left\{R_{j} \cup R_{k}\right\}=1-p\left\{\bar{R}_{j} \cup \bar{R}_{k}\right\} .
$$

As long as one of all sports service network communication nodes covers the grid element center $G\left(X_{j}, Y_{k}\right)$, the grid element center $G\left(X_{j}, Y_{k}\right)$ is considered to be covered, and the probability that the sports service network node covers the grid element center is the union of event $R_{i}$ [11], namely,

$$
p\left\{\bigcup_{i=1}^{N} R_{i}\right\}=1-p\left\{\bigcap_{i=1}^{N} \bar{R}_{i}\right\}
$$

where $N$ is the total number of communication nodes in the sports service network [12]. Then, the effective coverage rate of the communication node of the sports service network is

$$
P_{\text {cov }}=\sum_{j=1}^{m} \sum_{k=1}^{m} p\left\{\bigcup_{i=1}^{N} R_{i}\right\} \frac{\Delta S}{S} .
$$

\subsection{Energy Consumption Model of Sports Service Network} Nodes. According to the effective coverage rate of the sports service network node calculated above, the energy consumption model of the network node is constructed. The key to energy consumption of the sports service network's communication nodes is to transmit or receive data, calculate, and transfer data. The key link of energy consumption is network communication and mobile process [13]. For the analysis of the different states of the nodes, the mobile repeater represents the relay node of the communication node data of the sports service network, thereby minimizing the total energy consumption. The energy model uses the mobile scale distance consumption model as the energy consumed by the sports service network relay node during the movement, which is expressed as

$$
E_{M}(d)=P_{\text {cov }} k d \text {. }
$$

Among them, the energy consumed by the sports service network relay node $M$ to transfer the distance of $d$ is reflected as $E_{M}(d)$, and the $k$ parameter is determined according to the speed of the mobile communication network node's movement [14].

In the actual movement process, when $k=2$ is the optimal value, the data transmission energy consumption model is expressed as

$$
E_{T}(d)=m\left(a+b d^{2}\right) .
$$

Among them, the energy consumed to transmit the communication node $T$ of the sports service network to a location at a distance $d$ is denoted as $E_{T}(d)$. The size of the transmitted data packet is denoted as $m$, and the parameters $a$ and $b$ are determined due to environmental factors. Set all operations to be completed before the start of the mobile communication network data collection and transmission process. Set all mobile network nodes to determine their initial positions. The specific process is as follows:

The entire mobile communication sports service network is composed of several data aggregation nodes, a large number of sports service network relay nodes, and a large number of data collection nodes. The basic direction is based on the data summary node, and a direction routing tree is constructed [15]. At the same time, the sports service network relay node is introduced into the routing tree in this direction, and the topology of the routing tree is optimized to minimize the energy consumption of data transmission as much as possible. The following expressions define the entire mobile communication sports service network: $S=\left\{s_{1}, s_{2}, \ldots, s_{n}\right\}$ represents the set of $n$ nodes in the network, the $n$ positions where the sports service network can be deployed are denoted as $O=\left\{o_{1}, o_{2}, \ldots, o_{n}\right\}$, and $o_{i}$ represents the initial position of the node. The energy consumption of the entire mobile communication sports service network is mainly composed of the energy consumption generated during the node communication and the energy generated by the node transmitting data:

$$
(c\langle E, U\rangle c)=\sum E_{M}(d)+E_{T}(d) .
$$

Among them, $(c\langle E, U\rangle c)$ represents the total energy consumption of the sports service network, which mainly includes the following two parts: $E$ represents the energy consumption of the directional routing tree change node $S$ during the movement process and $U$ represents the energy consumption of the nodes at different locations in the process of transmitting network data. Total energy: through the above definition, the relevant symbols are brought into the above formula, and the following formula is used to express the energy consumption model of the communication node of the sports service network, indicating the total energy consumption during the change from the $i$ th node to the $j$ th node.

$$
c(\langle E, U\rangle)=\sum a m_{i} b m_{i}\left\|u_{i}-u_{j}\right\|^{2}+k\left\|o_{i}-u_{j}\right\| .
$$

\subsection{Optimization of Node Layout of Sports Service Network} Based on Accelerating Genetic Algorithm. The accelerated genetic algorithm is an improved algorithm of the standard genetic algorithm. Its core is to use the change space of the excellent individual variables generated by the previous evolutionary iterations as the initial change interval of the new variables and thus accelerate the cycle until the optimal individual optimization criterion function value. It is less than a certain set value or the algorithm runs to a predetermined number of acceleration cycles and ends. The algorithm steps can be summarized as follows:

Step 1: use binary coding, coding length is $e$, and the number of variables is $p$. After coding, the entire initial change space of the variable is discretized into $\left(2^{e}\right)^{p}$ grid points, and each grid point is regarded as an individual. 
Step 2: set the population size to $n$ randomly select $n$ points from the above $\left(2^{e}\right)^{p}$ grid points as the initial parent population.

Step 3: after decoding the code string of the parent individual obtained in the previous step, it is substituted into the optimization function to obtain the corresponding optimization function value. The smaller the value, the higher the fitness of the individual, and vice versa. Arrange the optimized function values in ascending order and define the top individuals as excellent individuals.

Step 4: probability selection of parent individuals, using proportional selection method, select the $G$ th individual from the original parent population with probability $g(i)$, and select a total of 2 groups, each with $n$ individuals.

Step 5: perform a two-point cross on the parent individual with the genetic crossover probability $p_{c}$. The larger the $p_{c}$, the faster the update of the code string in the population, and the stronger the search ability of the genetic algorithm.

Step 6: carry out two-point mutation on the offspring individuals with the genetic mutation rate $p_{m}$. Twopoint mutation is conducive to improving the diversity of the population, and $p_{m}$ represents the probability of individual mutation in the offspring.

Step 7: the $n$ offspring individuals obtained in Step 6 are used as new parents and then go to Step 3 to enter the next evolution process.

Step 8: speed up the cycle process. Take the change space of the excellent individual variables generated in the first and second evolutionary iterations as the initial change interval of the new variables and proceed to Step 1.

The optimization of the communication node layout of the sports service network is a multiobjective optimization problem. It not only achieves the maximum coverage of the sports service network communication nodes to the rectangular area but also minimizes the energy consumption of the network, so this article uses accelerated genetic algorithm to optimize the layout of sports service network nodes.

The optimization objective function of the node layout of the sports service network is designed as follows:

$$
f(x)=W_{1} \cdot f_{1}(x)+W_{2} \cdot f_{2}(x)
$$

The node layout optimization objective function is the weighted sum of two subobjective functions. In the formula: $W_{1}, W_{2}$ is the weighting factor, and the value of $W_{1}+W_{2}=$ 1 depends on the comprehensive requirements of the network designer for the performance indicators of the sports service network. To optimize the layout requirements of the sports service network nodes in this article, set the subobjective function $f_{1}(x)=P_{\text {cov }}, f_{2}(x)=1-J_{\text {net }}$. The two subobjective functions are transformed into the overall objective function by weighting, and they are used as the fitness function in the weighted genetic algorithm. The overall objective function can be defined as

$$
F_{\text {opt }}(x)=W_{1} \cdot P_{\text {cov }}+W_{2} \cdot\left(1-J_{\text {net }}\right) \text {, }
$$

where the two subobjective functions have been dimensionless and the function values are both $(0,1)$, and the total objective function value is also $(0,1)$. When $W_{1}$ is larger, the maximum coverage of the rectangular area of the sports service network communication node can be guaranteed; when $W_{2}$ is larger, the energy consumption of the sports service network communication node can be minimized. The larger the value of $F_{\text {opt }}(x)$, the better the scheme.

\section{Simulation Experiment Analysis}

3.1. Experimental Environment Settings. In order to verify the performance of the sports service network node layout optimization method proposed in this paper based on network communication in practical applications, a simulation experiment analysis was carried out in MATLAB 7.0 simulation software. Figure 1 shows the sports service network signal transmission platform:

Through the abovementioned sports service network signal transmission platform, the relevant parameters of the sports service network are set, and the test area size of the sports service network node is set to $120 \mathrm{~m} \times 120 \mathrm{~m}$, and each node is evenly distributed in the grid, the number of nodes is 150 , and the communication radius is $5-20 \mathrm{~m}$, the number of anchor nodes is 10-20, the simulation time is $100 \mathrm{~s}$, and the number of algorithm iterations is 400 . The method of literature [6], the method of literature [7], and this paper are used for comparative experiments.

3.2. Analysis on the Effect of Sports Service Network Node Layout. First, test the performance of the node layout of the sports service network of the method in this paper. The node layout results of the method in literature [6] are shown in Figure 2, the node layout results of the method in literature [7] are shown in Figure 3, and the optimized layout results of the method in this paper are shown in Figure 4.

In Figure 2, there is a large amount of overlap in the sports service network nodes laid out by the method of literature [6], and some areas are not fully covered. This phenomenon becomes more significant as the number of sports service network nodes increases. The effect of node layout is affected by the sports service network nodes. The influence of quantity is great; therefore, the method in literature [6] does not meet the requirements of the optimal layout of sports service network nodes.

In Figure 3, there is a small amount of overlap in the sports service network nodes laid out in the method proposed in [7], and some areas can be completely covered. The effect of the node layout is less affected by the number of sports service network nodes. Therefore, the method in literature [7] does not conform to requirements for 


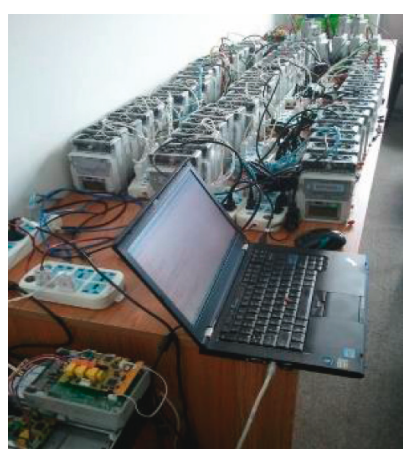

FIGURE 1: Sports service network signal transmission platform.

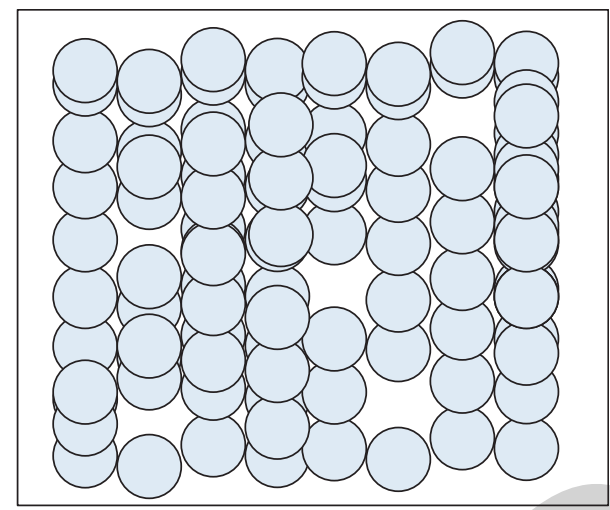

Figure 2: The layout effect of sports service network nodes based on the method of literature [6].

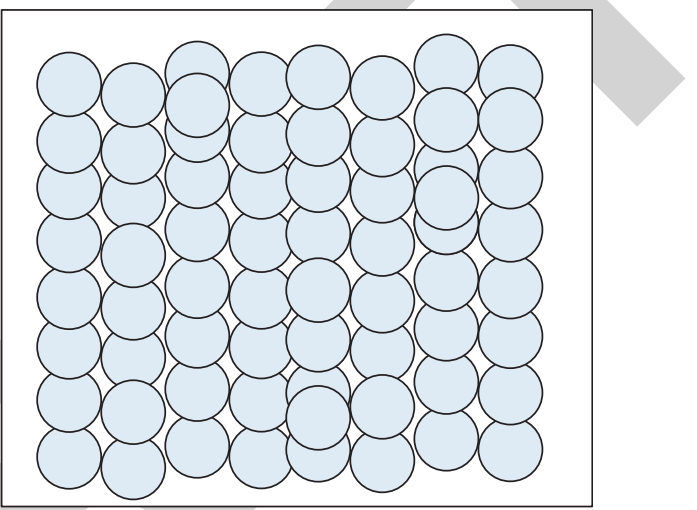

FIGURE 3: The layout effect of sports service network nodes based on the method in literature [7].

optimized layout of sports service network nodes. The node layout effect of the sports service network of the method in this paper is shown in Figure 4.

In Figure 4, the sports service network node layout optimization method based on network communication proposed in this paper is uniform, and there is no significant redundant node overlap phenomenon, which improves the layout effect of the method in [6]; when $N=100$, it basically achieves complete coverage of the detection area, the number of sports service network nodes continues to increase, and the optimized layout results are good. Therefore,

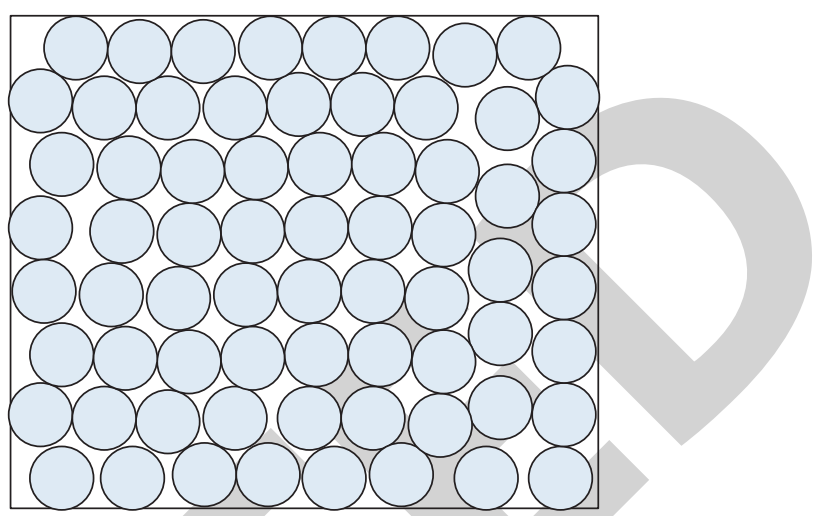

FIGURE 4: The node layout effect of the sports service network of the method in this paper.

the effect of the layout of the sports service network nodes in this paper is not affected by the number of sports service network nodes, and the results of the node layout are not interfered by the intermediate layout process.

3.3. Layout Node Performance Comparison. It evaluates the performance of the three methods of layout sports service network nodes from the coverage of the node layout method and the node moving distance and two aspects. Table 1 shows the coverage of the three methods for the layout of sports service network nodes, and Table 2 shows the movement distance of the nodes and the comparison.

In the test, the number of nodes in the transformed sports service network is $40,45,50,55,60,65,70,75,80$, and 85. The coverage rate is expressed in the form of a normalized value. The closer the result is to 1 , the stronger the coverage rate is. Analysis of Table 1 shows that, with the increase in the number of sports service network nodes, the coverage rate of the sports service network node layout optimization method based on network communication proposed in this paper gradually rises, and the coverage rate is in the range of 0.751 to 0.959 ; in the method of literature [6], the coverage rate is not stable, and the coverage rate is in the range of $0.358 \sim 0.578$; the coverage rate of the method in literature [7] is in the range of $0.523 \sim 0.686$. The coverage rate of the method in this paper is close to 1 and is stronger than the coverage rate of the other two methods. Node moving distance and comparison is shown in Table 2. In order to analyze the results more intuitively, corresponding bar charts are presented, and the results are shown in Figure 5 .

Combining Tables 1 and 2 to analyze the performance of different methods of laying out sports service network nodes, the continuous increase in the number of network nodes means an increase in the monitoring area of the sports service network, and the distance between nodes and nodes that need to be moved continues to increase. The method in [6] cannot reasonably allocate the movement of each sports service network node, which leads to confusion in the movement path and increases the total distance of the movement of the sports service network node. The total difference between the maximum monitoring range and the 
TABLE 1: Comparison of the coverage of the three methods.

\begin{tabular}{lccc}
\hline Number of network nodes/pieces & Method of this article & WSN [6] & SOP [7] \\
\hline 40 & 0.751 & 0.425 & 0.486 \\
45 & 0.789 & 0.358 & 0.523 \\
50 & 0.845 & 0.489 & 0.534 \\
55 & 0.862 & 0.578 \\
60 & 0.892 & 0.425 & 0.550 \\
65 & 0.912 & 0.514 & 0.561 \\
70 & 0.914 & 0.489 & 0.576 \\
75 & 0.925 & 0.543 & 0.582 \\
80 & 0.935 & 0.421 & 0.596 \\
85 & 0.959 & 0.630 \\
\hline
\end{tabular}

TABle 2: Node moving distance and comparison.

\begin{tabular}{lccc}
\hline Number of network nodes/pieces & Method of this article & WSN [6] & SOP [7] \\
\hline 40 & 215.3 & 289.3 & 315.5 \\
45 & 235.3 & 325.6 & 224.0 \\
50 & 245.6 & 356.4 & 278.5 \\
55 & 256.8 & 398.4 & 292.1 \\
60 & 287.9 & 443.2 & 347.2 \\
65 & 298.3 & 489.5 & 368.5 \\
70 & 312.3 & 526.2 & 465.4 \\
75 & 325.4 & 578.3 & 510.6 \\
80 & 358.9 & 596.1 & 523.4 \\
\hline
\end{tabular}

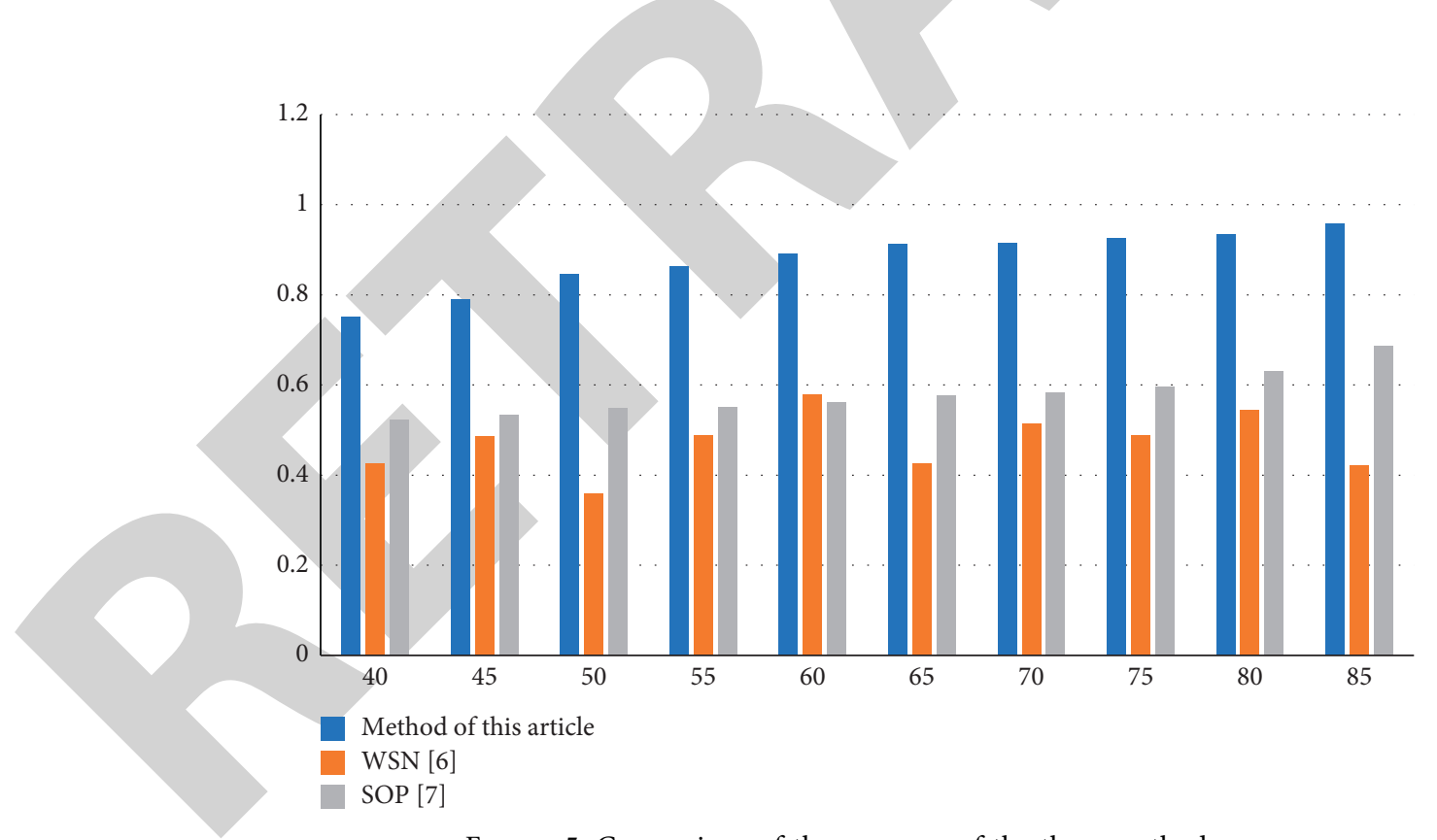

FIGURE 5: Comparison of the coverage of the three methods.

minimum monitoring range of the node distance reaches $306.8 \mathrm{~m}$ [7]. The layout effect of the method is also not ideal. The sum of the distances of the nodes moves is $332.7 \mathrm{~m}$; when the total number of sensors reaches the maximum of 85 , the sum of the distances of the nodes is only $378.6 \mathrm{~m}$, the maximum monitoring range and the minimum monitoring range. The total difference of the node distance is only $163.3 \mathrm{~m}$. In order to analyze the results more intuitively, corresponding bar charts are presented, and the results are shown in Figure 6. 


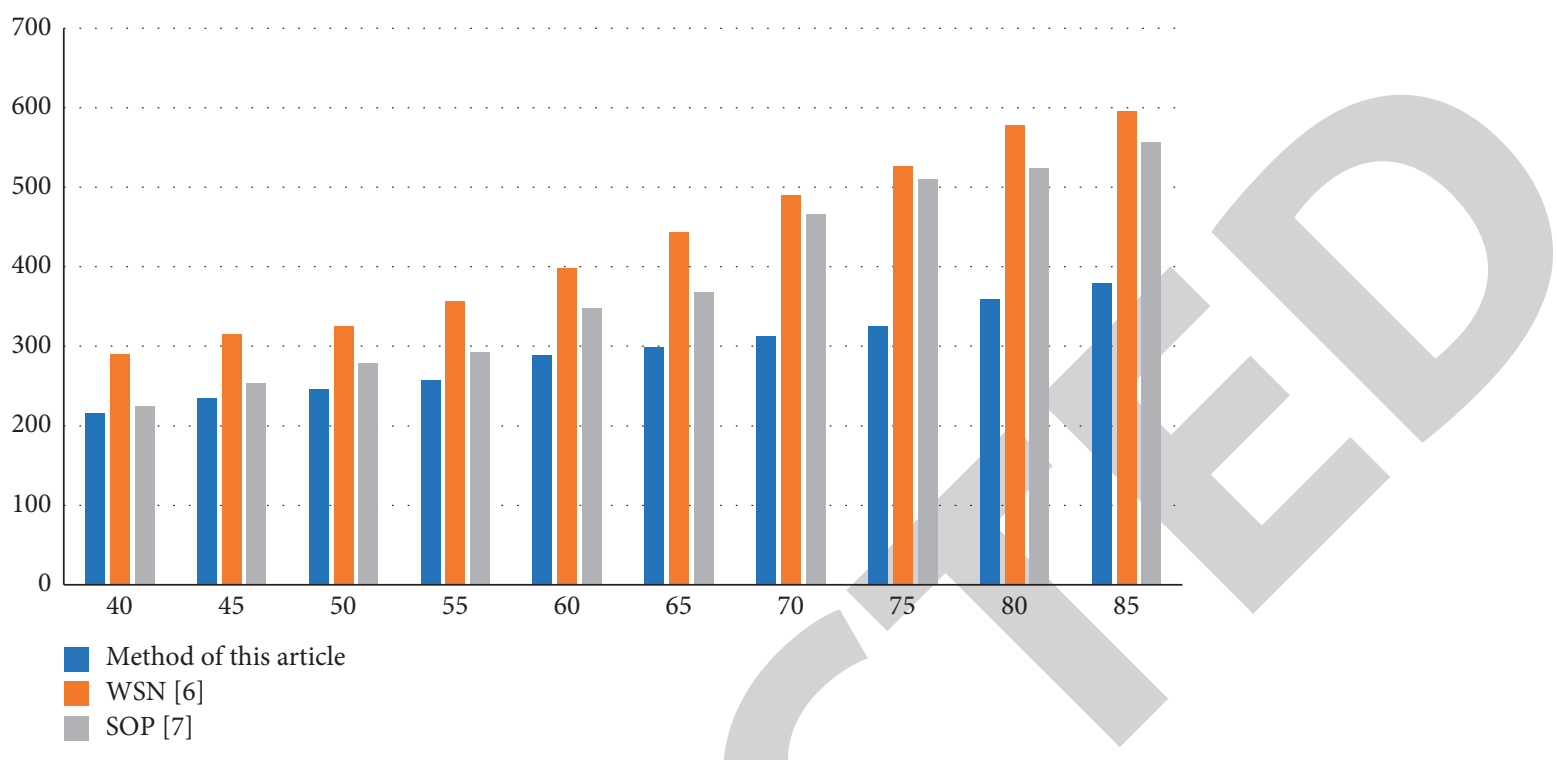

FIGURE 6: Node moving distance and comparison.

\section{Conclusion}

Faced with the problems of inefficient operation of stadiums, insufficient resource integration capabilities, and low levels of public services, building a sports service network platform has become an effective way to solve the above problems. The sports service network platform integrates modern network technology's high-efficiency, quickness, integration, and sharing characteristics. It is an important platform for the integration of stadium resources and an important link in the construction of sports informatization. It has played a huge role in public sports services and improving the operating efficiency of venues. In order to prolong the service life of the sports service network, optimizing the layout of sports service network nodes is a problem that needs to be solved urgently. For this reason, this paper proposes a method for optimizing the layout of sports service network nodes based on network communication. This method has a wide coverage of sports service network nodes. The node's moving distance is relatively short, effectively extending the life of the sports service network. Although our method has achieved good prediction accuracy at present compared with other popular methods, it is still unable to achieve considerable accuracy in the face of complex environment, and the training time of the model is long, which cannot meet the purpose of real-time predict. In the future, we will further optimize the model to improve the training speed while ensuring the accuracy.

\section{Data Availability}

The data used to support the findings of this study are available from the corresponding author upon request.

\section{Conflicts of Interest}

The authors declare that they have no conflicts of interest.

\section{References}

[1] J. Xie and J. Liu, "Simulation research on the optimization of wireless network communication coverage," Computer Simulation, vol. 8, no. 6, pp. 271-275, 2015.

[2] J. Han, S. He, M. Bi, and Z. Song, "Research on layout optimization of express parcel transportation network distribution center based on node operation process," Journal of Physics: Conference Series, vol. 1187, no. 4, pp. 042093-042103, 2019.

[3] Q. Yu, D. Yue, Y. Wang et al., "Optimization of ecological node layout and stability analysis of ecological network in desert oasis : a typical case study of ecological fragile zone located at Deng Kou County(Inner Mongolia)," Ecological Indicators, vol. 84, pp. 304-318, 2018.

[4] X. Peng and L. Xiong, "Multi-node communication channel optimization method based on hierarchical layout method," Electronic Design Engineering, vol. 27, no. 22, pp. 128-132, 2019.

[5] D. Shehab and H. Ammar, "Statistical detection of a panic behavior in crowded scenes," Machine Vision and Applications, vol. 30, no. 5, pp. 919-931, 2019.

[6] C. Yin, Y. Ye, L. Liang, and J. Yang, "Node layout optimization method of precipitation wireless sensor network in alpine watershed," System Engineering Theory and Practice, vol. 38, no. 8, pp. 266-274, 2018.

[7] Q. Liu, "Simulation of optimized positioning of congested nodes in mobile networks of the Internet of Things," Computer Simulation, vol. 35, no. 1, pp. 287-290, 2018.

[8] J. Wang, W. Wang, and W. Gao, "Fast and accurate action detection in videos with motion-centric attention model," IEEE Transactions on Circuits and Systems for Video Technology, vol. 36, no. 11, pp. 18-26, 2018.

[9] C. Xiang, "Optimal layout optimization of sensor network nodes based on distributed sound source positioning system," Automation Technology and Application, vol. 38, no. 10, pp. 74-77, 2019.

[10] C. Wang, A. Lu, and Y. Wu, "Application of adaptive fruit fly optimization algorithm in WSN node coverage optimization," Microelectronics \& Computer, vol. 36, no. 2, pp. 17-21, 2019. 
[11] D. Li, W. Yanting, and Y. Zhang, "Optimization strategy of WSN node distribution based on AVMC algorithm," $M i$ croelectronics \& Computer, vol. 36, no. 5, pp. 88-94, 2019.

[12] M. Gong and Y. Shu, "Real-time detection and motion recognition of human moving objects based on deep learning and multi-scale feature fusion in video," IEEE Access, vol. 41, no. 9, pp. 16-23, 2020.

[13] L. Wang, "Dynamic distributed node selection and optimization of ship vibration detection network," Ship Science and Technology, vol. 42, no. 4, pp. 158-160, 2020.

[14] R. Qiu, Y. Wang, and Z. Xue, "An optimized algorithm for I2P node selection against collusion attacks," Communication technology, vol. 53, no. 2, pp. 160-166, 2020.

[15] J.-U. Jung, Y.-K. Jeong, S. Ju, J. G. Shin, and J. Kim, “A simplified assessment method and application for consideration of survivability in spatial layout design at the early design stage of naval vessels," Journal of the Society of Naval Architects of Korea, vol. 55, no. 1, pp. 9-21, 2018. 